\title{
CS Research Square \\ A Qualitative Study on of Psychological Stress Among Frontline Nurses Fighting COVID-19
}

\section{Aihong Pan ( $\sim 3601409867 @ q q . c o m$ )}

Department of Nursing, The First People's Hospital of Hefei, Hefei City, China.

\section{Yuyan Wu}

The Binhu Hospital of Hefei

\section{Juanjuan Chen}

School of Nursing, Anhui Medical University

\section{Research Article}

Keywords: Qualitative Research, COVID-19, Frontline nurses, Psychological needs

Posted Date: February 1st, 2021

DOI: https://doi.org/10.21203/rs.3.rs-126454/v1

License: (1) This work is licensed under a Creative Commons Attribution 4.0 International License. Read Full License 


\section{Abstract}

Objectives To understand the mental health status of frontline nurses who were in direct contact with patients with confirmed or suspected coronavirus disease 2019 (COVID-19) and their tolerance for work duration and work intensity during the COVID epidemic, to identify the greatest source of stress, and to provide a scientific basis for targeted psychological interventions performed by clinical nursing managers to improve nurses' mental health.

Methods Using a purposive sampling method, a semistructured interview was conducted with 50 nurses working in the COVID ward, observation ward, and fever clinic of a COVID-19 designated treatment hospital in Hefei City. The interview data were sorted and analyzed using the Colaizzi phenomenological research method.

Results The frontline nurses felt stress, anxiety and loneliness, interpersonal distress, and psychological needs and had certain expectations regarding work duration and work intensity.

Conclusion Nursing managers should pay attention to the psychological needs of frontline nurses caring for COVID-19 patients, provide targeted intervention measures and make timely adjustments, and create a psychological stress response model for these nurses.

\section{1 | Introduction}

The coronavirus disease 2019 (COVID-19) epidemic has spread throughout China since January 2020, and various provinces and cities have initiated first-level response to major public health emergencies ${ }^{[1]}$. COVID-19 is contagious, and respiratory droplets and close contact are the main routes of transmission ${ }^{[2]}$. The rapid spread of COVID-19, its wide range of coverage, and its acute nature have put tremendous pressure on Chinese medical staff. The frontline nurses fighting the epidemic, that is, nurses who have direct contact with confirmed or suspected cases and are in close contact with patients, are usually under tremendous psychological pressure. Although the frontline nurses are carefully protected, they are still in a state of high-risk occupational exposure during various nursing operations. Most of them are in a state of high stress, with increased psychological pressure ${ }^{[3]}$. Studies ${ }^{[4]}$ have shown that among medical staff, as much as $60 \%$ may experience psychological crises in the event of an acute respiratory infection in the hospital. These conditions reduce the body's immunity and resistance to varying degrees and increase the chance of infection for nursing staff.

\section{2 | Aims And Objectives}

2.1 I Subjects Guided by descriptive phenomenological research method and using saturation of qualitative research data information as the standard, we used purposive sampling to enroll nurses who had worked for more than 2 weeks in the fever clinic, observation ward, or COVID ward of a COVID-19 designated hospital in Hefei as the subjects. Inclusion criteria were as follows: $\nabla$ Registered nurses who met the health industry standards of the People's Republic of China; $邓$ Nurses who worked during the 
epidemic and directly contacted suspected or confirmed cases; $\$ Those who signed informed consent to participate in this study voluntarily. Exclusion criteria were as follows: $₫$ Those who experienced major

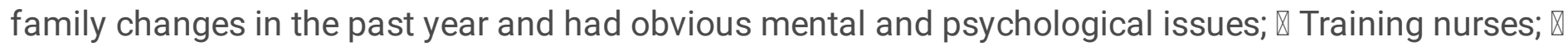
Those who could not understand the content of the interview. A total of 50 frontline nurses were interviewed. The subjects' names were replaced by "N1" to "N50" according to the order of the interviews. The general information is listed in Table 1.

\section{2 | Research methods}

2.2.1 Interview outline Based on the purpose of the study, a search of the relevant literature, the opinions of experts in infectious diseases and psychological consultation and experts with qualitative research background, and an interview with a COVID frontline nurse, the interview outline was created and revised by the study team. It included the following questions: (1) Do you have any anxiety (that affects your behavior, mood, cognition, or sleep status)? When did anxiety begin (duration of anxiety state)? When is your anxiety most obvious? Does this anxiety trouble you and have an effect on you? Do you want help to relieve your anxiety (or hope that your manager will take relevant measures)? (Record specific measures mentioned). (2) Do you like to express your feelings to others? Do you care about your current emotions? (3) Are you able to complete your own nursing work (competence at work, fatigue, and workload management)? Are you willing to communicate with doctors (reasonable division of roles between nurses and doctors)? (4) How long do you think is the most suitable continuous work time while wearing protective gear? What are your suggestions for nursing management?

2.2.2 | Materials Interview outline, informed consent, two recording pens, notebooks, and pens.

2.2.3 | Data collection method The phenomenological research method from qualitative research was adopted, and one-on-one interviews were conducted using a semistructured interview method. As the most commonly used method for collecting qualitative research data, semistructured interviews are relatively focused, do not deviate far from the topic, and can uncover a deep level of information ${ }^{[5]}$. An interview outline was developed before the interview, and the approval of the ethics committee was obtained. Before the start of the interview, we informed the interviewee of the purpose of the interview, summarized the related issues involved, and described the interview method and its meaning. Before the start of the interview, the participant signed an informed consent form. During the interview, we observed and recorded the expressions and other non-verbal behaviors of the interviewees, and we took notes and made recordings on-site, which were then archived ${ }^{[6]}$. The interviews were conducted when the interviewee had had sufficient rest, and night shifts and times when the interviewee was overworked were avoided to prevent psychological and emotional disturbances from affecting the interview results. Interviews were stopped when no new content was being added, and the interviews lasted 30 to 45 minutes ${ }^{[7]}$.

2.2.4 I Data analysis method The data analysis method was based on the basic principles of text transcription in qualitative research ${ }^{[8]}$. After each interview, the interview data were transcribed and stored 
within 24 hours. The content recorded during the interview and the interviewee's expression, gestures, movements, and body postures were noted in the corresponding places in the text.

2.2.5 Quality Control $₫$ An interview outline was developed before the interview, and the purpose of the interview was determined to avoid deviation from the subject. An appropriate interview time and place was chosen to facilitate a full understanding of the true thoughts of the interviewee. $\otimes$ The interviewer had a thorough understanding of qualitative research methods and interview skills, and we avoided having participants interviewed by their managers, which can cause tension for the interviewee. खDuring the interview, complex, abstract, or sensitive issues are discussed in the second half of the interview to allow the interview to proceed smoothly. $\otimes$ Before the formal interview, two frontline nurses were pre-interviewed to reduce the Hawthorne effect. $\triangle$ According to the rules for reaching consensus, two researchers transcribed and analyzed the interview content, and both parties needed to reach a consensus.

\section{3 | Results}

Through semistructured interviews with 9 frontline nurses, four themes were summarized: $\otimes f$ feeling tension, anxiety, and loneliness; 囚interpersonal disturbances; 『psychological needs; 『working environment, specifically expectations regarding working hours and work intensity.

\section{1 | Tension, anxiety, and loneliness}

3.1.1 There were 26 nurses who felt nervous and anxious about entering the frontline position in the fight against COVID-19. They were afraid that the disease would be highly contagious. In addition, they felt lonely because they were quarantined in a away from their families and children during their employment. Nurses 11, 23, 28, 39, 45, and 50 expressed the following: "I was with the first batch of nurses to enter the COVID-19 ward and have direct contact with patients. Because many procedures have not been determined, I don't know how to deal with patients. I feel nervous. The hospital isolation environment makes me feel anxious, and I can only fall asleep late at night." Nurses 2, 9, 12, 35, and 43 said, "I pay more attention to my family and children, and I use WeChat videos to call home every day...". Nurses 4, 44, 47, and 49 said, "I always help my son with homework every day. I'm not at home for the tutoring homework, and his father is not good at tutoring. Yesterday, the teacher criticized my son in the WeChat group. I called and reprimanded my son last night." Nurses 5, 8, 14 and 19 said, "I feel anxious these days. Maybe because of a change of environment, I don't sleep well at night and often wake up." Nurses 6, 17, 18 and 31 said, "I think I will feel anxious when I enter the isolation ward, especially for the night shift. I cannot wait for the end of the shift as soon as I enter the ward. I feel very tired when my work is particularly busy." Nurses 20,24 , and 29 said, "I saw on my phone that the epidemic is so serious, and so many people have died. I am worried about what my family will do if I get infected. So when I enter the isolation ward, I am very nervous and afraid of not wearing protective equipment in the right way. I ask my colleagues to help me repeatedly check whether the protective suit is torn."

3.1.2 Fear of being infected. Thirty-two nurses expressed worry about being infected. Nurses 4, 8, 10, 24, $28,34,37,46$ and 48 said, "For the three infected people admitted today, they just ate together at one 
meal. I feel that the COVID-19 is very contagious... we need to take protective measures. There was a patient who talks so close to me and coughs, and I am afraid that he is positive." Nurses $3,5,13,16,20$, $22,23,25,41$, and 43 said, "I feel that the medical surgical masks we are using are thinner than before. I am worried that they are not as protective as before. I am still afraid of being infected, even though the head nurse told us that although there is a shortage of supplies, the supplies provided to us are absolutely qualified." Nurses $14,17,30,35,41,44$, and 50 said, "When I atomize a patient or perform other nursing operations, the patient coughs, and I will worry for a long time and worry that I will be infected." Nurses $26,27,33,39,45$, and 49 said, "There is a patient who has repeatedly tested positive for nucleic acid. She can never get out of the hospital. I think she is highly infectious. Every time I perform a nursing procedure for her, I am always scared."

\subsection{3 | Physical discomfort caused by protective equipment Forty-one nurses reported that their physical} discomfort increased after wearing protective clothing and goggles. Nurses 5, 14, 26, 33, 37, 42, 47, and 49 said, "When I entered the isolation ward for the first time, I had the night shift. I felt a lack of air and then felt very sick and wanted to throw up, but fortunately, I had my colleagues." Nurses $8,10,18,20,23$, 28,34 , and 44 said, "It's very hard to breath. When I get busy, my body is all sweaty, and then I feel very cold when I have time to take a break." Nurses 2, 6, 15, 24, 35, and 39 all said in the interview, "After taking off the protective clothing and protective mask, I feel that the world is mine." Nurses 12, 21, 25, 40, and 46 said, "After putting on protective equipment, nursing procedures become more difficult. I almost can't place an intravenous infusion." Nurses 3, 7, 29, and 45 said, "I have become slower." Nurses 4, 11, 19, 32, 38, and 41 said, "The N95 mask makes me have an allergic reaction. No matter how good my skin care products are, they can't save me. The markings caused by the N95 mask won't go away." Nurses 9, 13, 17, and 27 said, "I'm okay. I mainly have hypoxia, and opening my mouth to breathe makes my mouth feel dry."

\subsection{Interpersonal relationship troubles In the interview, 18 nurses reported experiencing psychological} pressure in their relationships with colleagues and leaders. Nurses 5, 6, 14, 23, 33, and 44 said, "I'm not familiar with many colleagues and doctors here. I feel like I can't handle relationships with everyone, and I feel like there is no tacit understanding between us." Nurses $3,22,27,36,45$, and 49 said, "I think doctors go to the isolation ward less frequently and have less contact with patients every day. We go in the ward for six straight hours, which is a bit unfair." Nurses 14, 39 and 41 said, "The manager mentioned something a few days ago about changing the double shift to a single shift. I feel like I am more anxious working alone in the isolation ward with no one to talk with." Nurses 17, 30, and 32 said, "When I was working with a certain colleague, she always found excuses not to go to the ward, so I spent more time doing aerosol inhalation and measuring vital signs. I am worried that it will increase the risk of infection for me."

3.3 Psychological needs Thirty-one nurses felt that they needed psychological care. Nurses 12, 15, 23, $31,35,43$, and 50 said, "Communicating more with friends will make me feel happy, and my stress will be reduced a lot." Nurses 1, 9, 11, 16, 24, 29, and 33 said, "I video chat with my daughter every day, and talking to my daughter reduces my negative emotions." Nurses 3, 5, 13, 18, 37 and 46 said, "I used to feel 
anxious and become stressed easily, and sometimes it is difficult for me to adjust by myself...". Nurses $17,25,32,38,42$, and 47 said, "I think sometimes the care and encouragement of the manager is also very important. On the third day, when I had bad sleep and stressed, I had a conversation with the manager for a while and felt much better." Nurses 8, 19, 26, 39 and 49 said, "I sometimes feel like I need professional psychological counseling."

\section{4 | The longest working duration and the greatest work intensity that can be withstood In the interview,} 29 nurses indicated that they can work continuously for 6 hours at most, 11 nurses indicated that 8 hours of continuous work is acceptable, and 10 nurses indicated that the best uninterrupted work duration is 4 hours. Nurses 1, 4, 6, 39, 44, 46, and 47 said, "It's too stuffy to wear protective clothing. If I stay in the isolation ward and never come out, I think 6 hours is my limit. In terms of work intensity, if the patient's condition is mild, each nurse can manage about 10 patients." Nurses 2, 3, 12, 20, 30 said, "At the beginning of the epidemic, there were not enough people. My single shift lasted 12 hours in the ward. To save protective clothing, I didn't come out. I think 8 hours is acceptable now." Nurses 11, 13, 15, 17, 19, 22, 24, and 27 said, "Six hours is the most. If the patient needs intravenous infusion or sputum suction, I think I can only manage three patients at most." Nurses 7, 10, 21, 34, 35, and 38 said, "Six hours is the maximum, and after 6 hours, I will be a little irritable. As for the intensity of work, I have not encountered severely ill patients up to this point, and there is generally no problem with managing 9 patients." Nurses $33,40,41,42,49,50,23$, and 16 said, "Six hours is already my limit, and maybe I'm used to it...." Nurses $31,32,36,37,43$, and 45 said, "I receive an average of more than 70 patients per day. I think I can bear it for 8 hours." Nurses 5, 8, 9, 14, 18, 25, 28, 29, 26, and 48 said, "If I can't eat, drink water or use the toilet for 12 hours, I definitely won't be able to last. I think 6 hours is a bit too long. It's even better if a shift is only 4 hours."

\section{4 | Discussion}

\section{1 | Frontline nurses in the COVID ward and the observation ward have increased psychological pressure}

This study found through interviews that most of the frontline nurses had varying degrees of negative tension. The tension and loneliness of the frontline nurses in the COVID ward and the observation ward were most obvious due to the relatively closed working environment and the risk of infection. The highstress working environment is accompanied by excessive tension, anxiety, worry about themselves and their family members becoming ill, guilt related to their families, and even panic, restlessness, helplessness, and pessimism among medical staff, especially those on the front line fighting the epidemic. If these negative emotions are not addressed and resolved in a timely manner, they will have a substantial impact on individuals, hospitals, families, and society.

\subsection{Social and family support is vital to the frontline nurses fighting COVID-19. This study interviews} found that since the most of nurses were female, the most frequently mentioned concerns were about their children's lives and education. The second most frequently mentioned concern was the relationship with managers and colleagues. Family and social support play a very important role in relieving the stress 
of the frontline nurses of COVID-19. Research by Xu Ye et al. shows ${ }^{[12]}$ that the better an individual's family benefits are, the better the individual's job performance is. Nurses fighting COVID-19 need more family care and support to reduce the psychological pressure and burden of caring for COVID-19 patients.

4.3 | Nurses worry about being infected with COVID-19 COVID-19 is highly contagious and infects a large number of people, and there are currently no effective drugs for treatment. The number of patient deaths is increasing, and supplies are scarce during the epidemic. The interviewed nurses were worried that their occupational protection was not adequate. Most of the nurses worried about the risk of infection. The infection of frontline nurses will affect the mental state of other medical staff, who will then be unable to provide good nursing for patients. This suggests that nursing managers should provide frontline nurses with training on COVID-19 related knowledge and meet their demands for protective equipment in a timely manner.

\section{4 | The duration and intensity of work that frontline nurses can withstand while wearing protective} equipment The results of interviews with multiple frontline nurses show that the most suitable duration for uninterrupted use of protective equipment is 6 hours. Over 6 hours of wearing protective equipment can cause physical discomfort for the nursing staff, and their mental health may be affected. Wearing protective equipment increases the difficulty of performing nursing operations procedures and increases the nurses' workload. Under conditions requiring intravenous infusion, atomization inhalation, and sputum suction, one nurse can cover up to three patients. When these procedures are not required, one nurse can take care of ten patients.

\section{5 | Limitations of this study}

In summary, frontline nurses experience negative mental states such as tension, anxiety, and fear and need relevant support from society and their families to improve their mental health. Due to the nature of COVID-19, the comprehensive skills of nurses are in relatively high demand; most medical facilities require senior and experienced nurses, and most of the nurses are female. Therefore, our sample had certain limitations.

\section{5 | Intervention Measures}

5.1 To reduce the psychological stress of frontline nurses, our hospital invites nationally certified psychological counselors to provide psychological counseling through individual and group interventions. Individual interventions focus on interviewing; listening, understanding, and paying active attention to nurses after they enter the COVID-19 ward; positive resource utilization; relaxation techniques; mindfulness techniques ${ }^{[14]}$; group resource utilization and special periods and daily management based on the sunrise model of nursing ${ }^{[15]}$; the use of non-verbal resources in patient communication, ways to empathize with patients at special times, and the ability to ask for help; and how to gain more understanding and support from family members by expressing gratitude toward them. Group interventions take place during the rest times of nurses working in the COVID ward: first, the nurses are 
given an opportunity to talk to each other about their feelings and emotions; then, they are given time to talk about their own solutions and initiate discussions while receiving empathy, encouragement, support, and recognition of their hardship. Additionally, the group intervention allows nurses to see their own strength and their future self through narration, constructing signs of the physical behavior of COVID epidemic, energizing themselves and their peers, giving them tools for relaxation and meditation, and seeing themselves.

\section{2 | Application of traditional Chinese medicine (TCM) ear acupoint pressure using beans for} decompression intervention. Bean pressing of auricular points is a common TCM technique. Vaccaria segetalis seeds or rapeseeds can be placed at a tender point according to acupoint theory and then fixed to stimulate the acupoint or reaction point on the auricle. This technique can adjust the function of the viscera and the human endocrine system through the meridian system to achieve a balanced functional status ${ }^{[16]}$. According to a survey, the rate of ear acupoint pressure with beans in the Beijing area is as high as $89.3 \%$, and this method was effective for treating constipation, insomnia, and headaches ${ }^{[17-20]}$. In this study, teachers from the Chinese traditional medicine nursing clinic were invited to perform ear acupoint pressure with beans on the frontline nurses to reduce and relieve their anxiety.

5.3 I Implement protective measures. We should ensure the supply and quality of protective equipment for frontline nurses, provide rest places during work, and ensure that they are consuming an adequate diet and nutrition, which should be overseen by special personnel. We should also arrange reasonable shifts, solicit opinions from those involved in real time, and make prompt adjustments. We need to encourage treatment and nursing staff to communicate with each other and provide timely feedback on problems to meet their needs.

\section{Declarations}

Authors' contributions: All authors (Aihong Pan, Yuyan Wu, and Juanjuan Chen) have participated in the concept, design, analysis and/or interpretation of these data, drafting of the manuscript, and assume responsibility for the research. Everyone was involved in revising the manuscript critically for important intellectual content. Everyone made substantial contributions to the conception and design, acquisition of data, and analysis and interpretation of data. The author(s) read and approved the final manuscript.

Availability of data and materials: All data generated or analysed during this study are included in this published article [and its supplementary material files].

Funding Statement: This project is financed by the Hefei Municipal Health Commission on the construction of scientific research projects for the prevention and control of the coronavirus disease epidemic in 2020. Project number is Hwk2020yb001. The funder was not involved in the study design and will not contribute to data collection, analysis, interpretation of data or manuscript writing and the decision to submit the report for publication and will not have ultimate authority over any of these activities. 
Conflict of Interest statement: The authors have no conflicts of interest to disclose.

Ethics approval and consent to participate: The study was approved by the Ethics Committee of The First People's Hospital of Hefei, China (Approval No. 2020-010-01).

Ethical Declarations $\square A l l$ methods were carried out in accordance with the relevant guidelines and regulations.

Animals Ethical Approval: This article does not contain any studies with animals performed by any of the authors.

Informed Consent: Written informed consent was obtained from all individual participants included in the study.

Consent for publication: Not applicable.

\section{References}

1. Lu Yalan, Liu Cong, Zhou Wenzheng, et al. Epidemiological Characteristics of COVID-19, SARS and MERS and Their Prevention and Control Measures. Herald of Medicine: 1-13.

2. Notice on Issuing the Novel Coronavirus Pneumonia Diagnosis and Treatment Plan (Trial Version 7). General Office of the National Health Commission of the PRC. National Health Commission Official Letter. [2020] No. 184.

3. Lu Y, Jia YR, and Gao FL. A study review of the psychological status of health care professionals engaged in emerging acute respiratory infectious diseases. Chinese Nursing Management. 2019;19(1):83-86.

4. Low JG, Wild-Smith A. Infectious respiratory illnesses and their impact on health care workers: a review. Ann Acad Medsingapore. 2005; (34):105-110.

5. Yin L.Semi-structured interview. Chinese Nursing Management. 2019;19(5):783.

6. Lu Y, Jia YR, Gao FL. A qualitative study on the psychological experience of patients with interstitial lung disease. Nursing Research. 2020; 34(6):1089-1091.

7. Y LL, Gao LN, Dai YQ, et al. A qualitative study on the inner experience of frontline nurses in the face of patient death during the fight against novel coronavirus pneumonia. Chinese General Practice Nursing. 2020; 18(10):1208-1211.

8. Li Z, and Liu Y. Nursing research methods. Beijing: People's Medical Publishing House; 2019.

9. Li WH. Protection and psychological counseling of the public in the face of novel coronavirus pneumonia. Changsha: Central South University Press; 2020; 83-115.

10. Zhu YM, Zhang Y, and Liu DL. Relationships between social support, mental health level and subjective well-being in general surgical nurses with different nursing age. Occupation and Health. 2016; 32(7): 916-920,924. 
11. Shen XH, Sun J, Guo Y, et al. A Survey of the Comprehensive Well-being of Medical Staff. Modern Hospital. 2019; 19(8): 1093-1098.

12. Xu Y, Bao WT, and Li QF. Research on the impact of nurses' social support and work-family gain on subjective well-being. Today Nurse. 2020; 29(6): 26-29.

13. Mei L, Mei P, and Li Q. Investigation of the psychological status and coping styles of the first batch of frontline medical staff facing the novel coronavirus pneumonia epidemic. Journal of Qilu Nursing. 2020; 26(13): 40-43.

14. He YQ, and Hua YL. Mindfulness Meditation from the Perspective of Psychology. Journal of Wuhu Vocational and Technical College. 2016; 18(1): 14-16.

15. He GP, and Yu J. Practical Nursing. Beijing: People's Medical Publishing House; 2002.

16. Zhang XY, Ma JY, Li XY, et al. Analysis of research hotspots for the traditional Chinese medicine nursing technology of ear acupoint pressure with beans based on co-word analysis. Shanghai Nursing. 2019;19(11): 37-41.

17. Ma XB. Investigation of the status quo of traditional Chinese medicine nursing health education and technical operation in Beijing Hospital of Traditional Chinese Medicine. Beijing: Beijing University of Chinese Medicine; 2012.

18. Luo CM, Wang J, Chen Y, et al. Effects of Ear Acupressure in treatment of constipation: a systematic review. Chinese Nursing Management. 2017;17(4): 548-554.

19. Zhang J, Lu JP. Therapeutic efficacy of auricular point pressing with beans combined with Chinese medicine foot bath in insomnia patients. Shanghai Nursing. 2017;17(3):34-36.

20. Fu GL, Sun LH, and Liu XX. Research development on the treatment of migraine with bean-pressing on auricular points. Chinese Journal of Modern Nursing. 2017; 23(35): 4554-4556.

\section{Tables}

Table 1 General information of the subjects $(n=50)$ 


\begin{tabular}{|c|c|c|c|c|c|c|}
\hline Number & $\begin{array}{l}\text { Age } \\
\text { (years) }\end{array}$ & Gender & $\begin{array}{l}\text { Marital } \\
\text { status }\end{array}$ & Education & Title & $\begin{array}{l}\text { Work experience } \\
\text { (years) }\end{array}$ \\
\hline N1 & 31 & Female & Married & Bachelor & Nurse practitioner & 9 \\
\hline N2 & 29 & Female & Married & Bachelor & Nurse practitioner & 9 \\
\hline N3 & 36 & Male & Married & $\begin{array}{l}\text { Master } \\
\text { student }\end{array}$ & $\begin{array}{l}\text { Charge nurse } \\
\text { practitioner }\end{array}$ & 12 \\
\hline N4 & 38 & Female & Married & Bachelor & $\begin{array}{l}\text { Charge nurse } \\
\text { practitioner }\end{array}$ & 15 \\
\hline N5 & 34 & Female & Married & Bachelor & $\begin{array}{l}\text { Charge nurse } \\
\text { practitioner }\end{array}$ & 12 \\
\hline N6 & 32 & Female & Married & Bachelor & Nurse practitioner & 10 \\
\hline N7 & 31 & Female & Married & Bachelor & Nurse practitioner & 11 \\
\hline N8 & 31 & Female & Married & Bachelor & Nurse practitioner & 10 \\
\hline N9 & 30 & Female & Married & Bachelor & Nurse practitioner & 11 \\
\hline N10 & 35 & Female & Married & Bachelor & $\begin{array}{l}\text { Charge nurse } \\
\text { practitioner }\end{array}$ & 12 \\
\hline N11 & 32 & Female & Married & Bachelor & Nurse practitioner & 11 \\
\hline N12 & 31 & Female & Married & Bachelor & Nurse practitioner & 10 \\
\hline N13 & 34 & Female & Married & Bachelor & Nurse practitioner & 12 \\
\hline N14 & 27 & Female & Single & Bachelor & Nurse practitioner & 10 \\
\hline N15 & 34 & Female & Married & Bachelor & Nurse practitioner & 11 \\
\hline N16 & 36 & Female & Married & Bachelor & Nurse practitioner & 13 \\
\hline N17 & 38 & Female & Married & Bachelor & $\begin{array}{l}\text { Charge nurse } \\
\text { practitioner }\end{array}$ & 18 \\
\hline N18 & 30 & Female & Married & Bachelor & Nurse practitioner & 11 \\
\hline N19 & 34 & Female & Married & Bachelor & Nurse practitioner & 10 \\
\hline N20 & 33 & Female & Married & Bachelor & Nurse practitioner & 9 \\
\hline N21 & 26 & Female & Single & Associate & Nurse practitioner & 4 \\
\hline N22 & 29 & Female & Single & Bachelor & Nurse practitioner & 7 \\
\hline
\end{tabular}




\begin{tabular}{|c|c|c|c|c|c|c|}
\hline N23 & 34 & Female & Married & Bachelor & $\begin{array}{l}\text { Charge nurse } \\
\text { practitioner }\end{array}$ & 11 \\
\hline N24 & 31 & Female & Married & Bachelor & Nurse practitioner & 9 \\
\hline N25 & 31 & Female & Married & Bachelor & Nurse practitioner & 9 \\
\hline N26 & 35 & Female & Married & Bachelor & Nurse practitioner & 12 \\
\hline N27 & 35 & Female & Married & Bachelor & Nurse practitioner & 13 \\
\hline N28 & 35 & Female & Married & Bachelor & Nurse practitioner & 14 \\
\hline N29 & 38 & Female & Married & Bachelor & $\begin{array}{l}\text { Charge nurse } \\
\text { practitioner }\end{array}$ & 18 \\
\hline N30 & 26 & Female & Married & Associate & Nurse practitioner & 8 \\
\hline N31 & 32 & Female & Married & Bachelor & Nurse practitioner & 8 \\
\hline N32 & 32 & Female & Married & Bachelor & Nurse practitioner & 8 \\
\hline N33 & 35 & Female & Married & Bachelor & $\begin{array}{l}\text { Charge nurse } \\
\text { practitioner }\end{array}$ & 12 \\
\hline N34 & 32 & Female & Married & Bachelor & Nurse practitioner & 11 \\
\hline N35 & 22 & Female & Single & Bachelor & Nurse practitioner & 2 \\
\hline N36 & 34 & Female & Married & Bachelor & Nurse practitioner & 13 \\
\hline N37 & 27 & Female & Single & Bachelor & Nurse & 5 \\
\hline N38 & 33 & Female & Married & Bachelor & Nurse practitioner & 10 \\
\hline N39 & 34 & Female & Married & Bachelor & Nurse practitioner & 11 \\
\hline N40 & 31 & Female & Married & Bachelor & Nurse practitioner & 9 \\
\hline N41 & 28 & Female & Single & Bachelor & Nurse practitioner & 8 \\
\hline N42 & 26 & Female & Single & Associate & Nurse practitioner & 5 \\
\hline N43 & 33 & Female & Married & Associate & Nurse practitioner & 11 \\
\hline N44 & 34 & Female & Married & Bachelor & Nurse practitioner & 12 \\
\hline N45 & 30 & Female & Married & Bachelor & Nurse practitioner & 9 \\
\hline N46 & 33 & Female & Married & Associate & Nurse practitioner & 10 \\
\hline N47 & 34 & Female & Married & Bachelor & Nurse practitioner & 12 \\
\hline N48 & 22 & Male & Single & Associate & Nurse & 2 \\
\hline N49 & 36 & Female & Married & Bachelor & Nurse practitioner & 14 \\
\hline
\end{tabular}


